\title{
Self Reported Dental Health, BMI, and Albumin in a Geriatric Population
}

\author{
M. Midttun, Z. Zahir \\ Department of Geriatrics, University Hospital Copenhagen Hvidovre, Kettegaard Allé 30, Hvidovre, Denmark \\ Email: info@mettemidttun.dk, zebazaher@hotmail.com
}

Received 3 March 2014; revised 4 April 2014; accepted 30 April 2014

Copyright $@ 2014$ by authors and Scientific Research Publishing Inc.

This work is licensed under the Creative Commons Attribution International License (CC BY). http://creativecommons.org/licenses/by/4.0/

(c) (i) Open Access

\begin{abstract}
Method: 99 patients, 70 women and 29 men, mean age 84.2, were included. They were all hospitalized in the geriatric department between June and September 2010. The study was a questionnaire, and the questions were read out loud by one of the investigators. The answers were the patient's own experience. The patient's mouth and teeth were not examined. BMI and albumin were taken from the patient's medical record. Results: There was no statistical difference in albumin (mean: 31.2, 22.5) or BMI (mean: 29.3, 23.8) between patients with natural teeth, and a denture, ( $p=0.12$, and 0.23), but mean albumin was slightly higher in patients with natural teeth. Patients with a denture were significantly older than patients with natural teeth $(p=0.02)$. Conclusion: In general the patients were happy with their teeth even though $71.7 \%$ had a denture, and $44.1 \%$ said that it caused problems. All patients with natural teeth except one consulted the dentist frequently. 15\% answered that they had bad dental health, but there was no significant difference in BMI and albumin between the groups. Still it is of great importance to bear in mind that when dealing with patients with digestive problems, malnutrition, infections, wounds etc. the fact that these symptoms could possibly be caused by a poor dental state should be considered, especially in geriatric patients, a group of patients that are still getting older.
\end{abstract}

\section{Keywords}

Dental Health, Teeth, Oral Health, Nutrition, Geriatric Patients, Oldest Old, BMI, Albumin

\section{Introduction}

Sufficient nutrition is of great importance for as well general as oral health, especially in the elderly. And oral health and comfort are prerequisites for a good masticatory function and adequate food intake [1]. It is well known that poor oral health and xerostomia are frequently associated with a reduced BMI (body mass index), a 
low serum albumin level, and the avoidance of difficult-to-chew foods. Nevertheless very little interest is taken in the patient's dental state when they are hospitalized [1].

Most studies of dental health in the elderly are made in nursing homes. In general very little data exist on the oldest old, and the patient's own experience of his or her dental health is usually not taken into account. The importance of a good dental health has been described in various studies, and generally the relations between tooth loss and the phycho-social well being, general health, chewing efficiency, digestive problems, and mortality are well documented but the underlying mechanisms are not fully understood [1]-[5]. It has been shown that oral care intervention in elderly in a nursing home kept the patients nutritional state stable during the year of the study, whereas all indicators declined in the intervention group that did not receive dental care [3]. It has been shown that among the frail, institutionalized patients examined, those with compromised oral functional status had the significantly lowest BMI and serum albumin concentrations, and those who were seen regularly by a dentist showed no decline in nutritional status in contrary to the patients that did not receive regular oral control [3] [6]. A study from Taiwan showed that self-reported unsatisfactory chewing ability was associated with a greater mortality risk in older adults when coexisted with metabolic syndrome [7].

One single American study exists where a model developed to calibrate and adjust self-reported oral status in a large population was introduced [8]. To our knowledge no recent studies have been made where the relation between the patient's own experience of his or her dental health and objective data as BMI and albumin as an expression of nutritional state were examined in geriatric patients. It was therefore decided through a small questionnaire to examine hospitalized geriatric patient's self reported dental state and compare the results to BMI and albumin.

\section{Material and Method}

99 patients, 70 women and 29 men, mean age 84.0 years, median age 84.2 (66 - 102) years were included. They were all hospitalized in the geriatric department of Copenhagen University Hospital Hvidovre, situated just outside the center of Copenhagen, the capital of Denmark, between June and September 2010. The patients included were all home dwelling, middle-class citizens that were hospitalized due to an acute decline in their functional state because of infection, dehydration, electrolyte derangement etc., and when at home they all have access to daily public help when needed. All patients gave their informed consent. The study was a pilot a study made in order to get an impression of the number of patients needed to include in a later and bigger study. It was decided to include 100 subjects, a number that seemed realistic according to the time available for the project. All patients hospitalized in the period were asked and accepted to participate. One patient was included twice, and the second questionnaire was therefore excluded. The study was made as a questionnaire consisting of seven short questions, and 2 - 3 answering possibilities that in general could be answered in monosyllables, see Figure 1 . The questions were read out loud by one of the two investigators. All answers were the patient's self reported experience, and the patient's mouth and teeth were not examined. Furthermore the patient's weight, height, age, sex, BMI, and albumin were filled in by one of the investigators. These results were taken from the patient's medical record.

The statistic calculations were made by a local statistician. Age and BMI were analyzed by one-way ANOVA procedure, whereas distribution of sex was compared by the chi-square test. Level of significance for all testes was 0.05 .

\section{Results}

The results showed that 71 patients (71.7\%) had a denture, 29 of these patients had partial denture, 42 had full denture, 26 patients had natural teeth, and 2 patients had no natural teeth and no denture were edentulous. 29 of these patients (40.8\%) mentioned problems with their denture, and 18 patients (44.1\% of these patients) complained that their denture fitted badly. $15.2 \%$ (15 patients) thought that their dental health was poor, the rest thought that their dental health was good (61 patients) or acceptable (22 patients), one did not know. 77.7\% never experienced toothache, $17.2 \%$ had toothache now and then, and two patients had constant pain. $60 \%$ of the patients were between 80 and 90 years old, and 32\% (19 of these patients) had natural teeth. 21 patients were $90+$ years old, and one was 102. Among the 90+ years old, 3 patients had natural teeth, and the 102 years old patient had a denture. All patients but one with natural teeth consulted a dentist regularly. See Figure 2. 


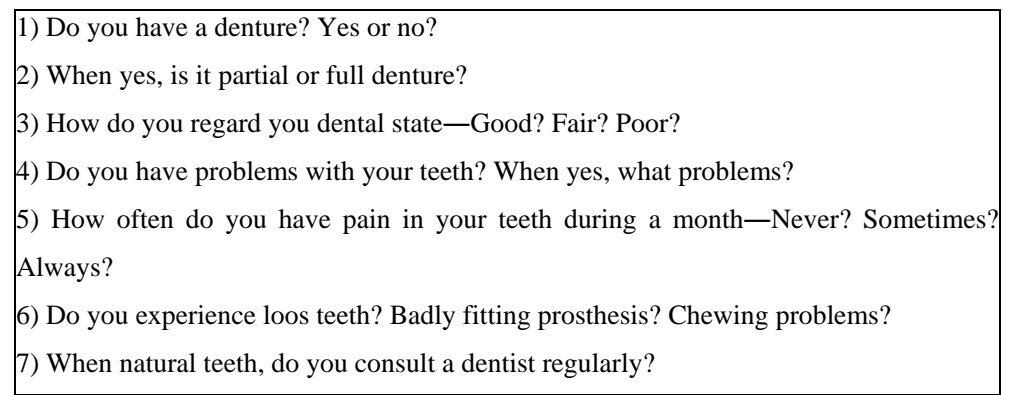

Figure 1. Questionnaire translated into English.

\begin{tabular}{|c|c|c|c|c|c|c|c|c|c|c|}
\hline $\begin{array}{c}\text { Natural } \\
\text { teeth }\end{array}$ & Denture & $\begin{array}{c}\text { Full } \\
\text { denture }\end{array}$ & $\begin{array}{c}\text { Partial } \\
\text { denture }\end{array}$ & No teeth & $\begin{array}{c}\text { Good } \\
\text { dental } \\
\text { health }\end{array}$ & $\begin{array}{c}\text { Acceptable } \\
\text { dental } \\
\text { health }\end{array}$ & $\begin{array}{c}\text { Bad } \\
\text { dental } \\
\text { health }\end{array}$ & $\begin{array}{c}\text { Never } \\
\text { toothache }\end{array}$ & $\begin{array}{c}\text { Now and } \\
\text { then } \\
\text { toothache }\end{array}$ & $\begin{array}{c}\text { Chronic } \\
\text { pain }\end{array}$ \\
\hline 26 & 71 & 42 & 29 & 2 & 61 & 22 & 15 & 77 & 17 & 2 \\
\hline
\end{tabular}

71 patients had a denture, 29 of these patients mentioned problems with their teeth, and 18 due to bad fitting.

Figure 2. Number of patients in the different groups.

There was no statistical difference in albumin (mean: 31.2, 22.5) or BMI (mean: 29.3, 23.8) between patients with natural teeth, and a denture, $(\mathrm{p}=0.12$, and 0.23$)$, but mean albumin was slightly higher in patients with natural teeth. There was no significant difference in sex between patients with denture and natural teeth. Patients with a denture were significantly older than patients with natural teeth $(p=0.02)$, Figure 3 and Figure 4.

\section{Discussion}

When hospitalized the patients are usually asked whether they have a denture or natural teeth, but dental problems are generally not paid much interest in the hospital even though it is well known that dental health is of great importance as dental problems may cause digestive problems, malnutrition, infections, wounds, etc., symptoms that are all frequently represented in the geriatric patients. Furthermore the fact that various types of medicine may lead to dental problems are often overlooked [1] [2].

In the present study we emphasize the importance of describing the patients own experience of his or her dental state, and compare the answers to serum albumin and BMI. In previous studies where the patients teeth were examined by a dentist or a dental hygienist, lower BMI and albumin were found in subjects with a compromised oral status, and the presence of fewer than six occluding pairs of teeth was one of the two best predictors of malnutrition, whereas in edentulous subjects the absence of dentures was strongly associated with a low BMI. Those who have more than 20 natural teeth left reported no chewing problems [1]. However, neither BMI nor albumin was lower in the edentulous than in the dentate group in a study made by Mojon et al. in 1999 [4]. In the present study only two edentulous patients were included, and there was not found any significant difference in albumin or BMI between the groups examined, only mean albumin seemed to be slightly lower in the patients with poor teeth. The reason why most of the patients in the present study have BMI and serum albumin within normal limits may be that they are in a generally good shape and able to compensate for their poor dental state by e.g. nutrition drinks. Previous studies have shown that life expectancy of edentulous persons is lower than in those who retain at least some teeth until late in life, and some studies have shown an association between mortality in people aged 70, and chewing ability [2] [7]. The poorer the general health the less natural teeth are present, and a study on community-dwelling elderly persons revealed that cognitive decline is related to few or no natural teeth [2] [5].

In general dental health has improved dramatically over the past decades [9]. In Sweden, a society that is comparable to the Danish society, the number of edentulous citizens between 40 and 70 years old was reduced from $16 \%$ in 1979 to $1 \%$ in 2003 [9]. Danish figures from the same period showed equal results [10]. In the beginning of the 1980's almost 60\% between 65 - 81 years were edentulous, and only 17\% of these patients had a sufficient number of teeth and could manage without a denture [10]. As early as in 1994 these figures had improved dramatically as $60 \%$ at the age of 65 - 74 had natural teeth and among those above 75 years old, $45 \%$ had natural teeth. This tendency seems to continue [10]. Very little data exist on the oldest old, but a German 


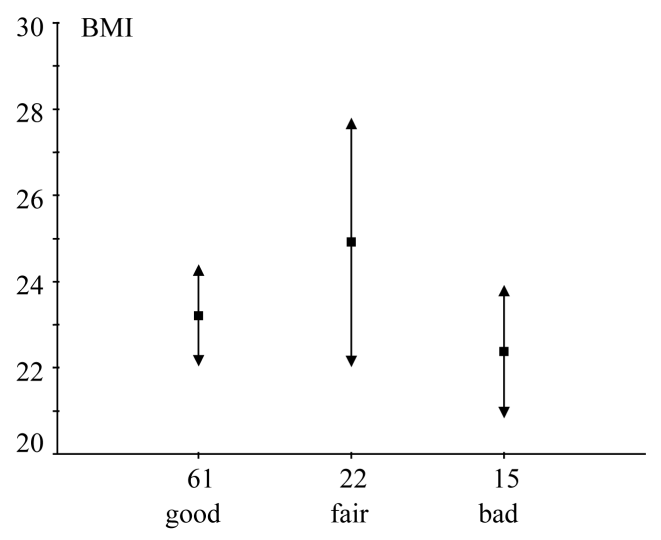

Figure 3. Graphic presentation of BMI as a function of self-reported dental health. Squares denote mean BMI and arrows mark the 95\% confidence interval for the means.

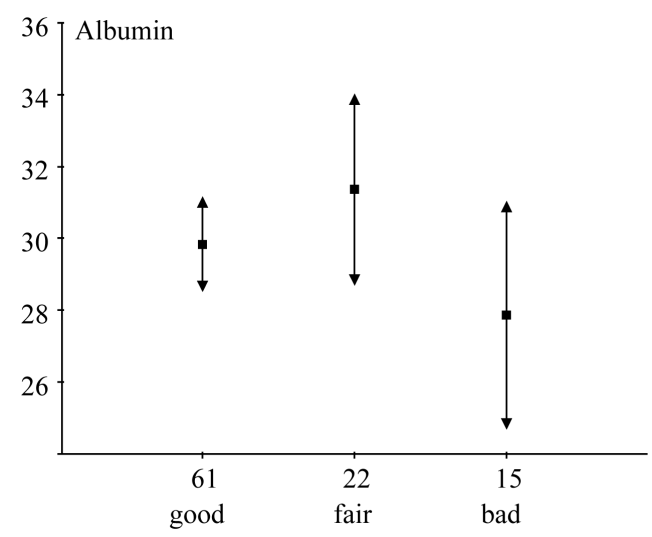

Figure 4. Albumin as a function of self-reported dental health. Squares denote mean albumin (g/l), and arrows mark the 95\% confidence interval for the means.

study from 1996 showed that $40 \%$ of the 80 - 84 old persons, and $75 \%$ of the 90 - 94 year old persons were edentulous [2]. In the present study where the patients mean age was 84.2 years, 21 patients were $90+$ years old, 3 of these had natural teeth. One patient was 102 years old and had a denture. $70.7 \%$ of all patients included were edentulous for practical reasons, but only two patients had neither a denture nor natural teeth.

In the present study $40.8 \%$ complained of problems with their denture, and two of these patients had more serious problem as they admitted constant pain when questioned. $44.1 \%$ (18 of these patients) complained of a badly fitting prosthesis that may cause discomfort and thereby prevent the patient from chewing the food properly with the complications that follow [1] [2] [5]. The chewing efficiency is closely related to the condition of the teeth, and chewing problems may give rise to digestive symptoms, most probably because of the larger particles of swallowed food [1]. A solution may be a well-made dental prosthesis that can easily be obtained by the placement of implants to retain the denture that may play a key role in limiting morbidity and supporting successful aging, and usually a considerable improvement in chewing ability [1] [2].

The present results confirm previous studies showing that still many old patients tend to wear an old denture that fit poorly, but they are rarely motivated or have the strength to seek and benefit from prosthodontic treatment [1]. Many of these problems could easily be dealt with if the hospitals in general had access to a hygienist or a dentist. Even though the old and the oldest old are home dwelling and well enough to live in their own home, they frequently do not have the strength to contact a dentist on their own, not everybody have relatives to help them solve this problem, and many do not tell anybody about their problems unless they are asked directly. 
Therefore their teeth (denture) problems are not revealed until they for some other reason go to the hospital, and in the hospital the dental problems are not taken care of. A similar conclusion was made in a Swiss study from 1995 [11].

This study includes a relatively large number of elderly, but still more patient should be included in future study as 99 are too little to obtain significant results. All the patients included are citizens from the same environment, and a more mixed population would be preferable in a future study. The old are still getting older, and consequently more patients above 90 years should be included. Furthermore it would be desirable to make at bigger study where the patients teeth were examined by a dentist or perhaps a dental hygienist. The patients included in the present study were very willing to talk about their dental health, so most probably a more thorough interview and examination would be accepted by the patients.

\section{Conclusion}

In general, the 99 patients included in the study were very content with their teeth even though $71.7 \%$ had a denture, and $44.1 \%$ of these patients admitted that it caused problems. Furthermore the patients with natural teeth took very good care of their teeth and went to see the dentist regularly in spite of their generally reduced physical strength. 15\% complained of poor dental health, but there was no significant difference between BMI and albumin in the present study, and only mean albumin had a tendency of being lower in those with poor teeth. When dealing with patients with digestive problems, malnutrition, infections, wounds etc. the fact that these symptoms could possibly be caused by a poor dental state should be considered, especially in geriatric patients, a group of patients that are still getting older.

\section{References}

[1] Budtz-Jørgensen, E., Chung, J.P. and Rapin, C.H. (2001) Nutrition and Oral Health. Best Practice and Research. Clinical Gastroenterology, 15, 885-896.

[2] Müller, F. and Schimmel, M. (2010) Tooth Loss and Dental Prostheses in the Oldest Old. European Geriatric Medicine, 1, 239-243. http://dx.doi.org/10.1016/j.eurger.2010.06.001

[3] Sumi, Y., Ozawa, N., Miura, H.K., Michiwaki, Y., et al. (2009) O. Oral Care Helps to Maintain Nutritional Status in Frail Older People. Archives of Gerontology and Geriatrics, 51, 125-128. http://dx.doi.org/10.1016/j.archger.2009.09.038

[4] Furuta, M., Komiya-Nonaka, M., Akifusa, S., et al. (2012) Interrelationship of Oral Health Status, Swallowing Function, Nutritional Status, and Cognitive Ability with Activities of Daily Living in Japanese Elderly People Receiving Home Care Service Due to Physical Disabilities. Community Dentistry and Oral Epidemiology, 41, 173-181.

[5] Torres Luisa Helena do Nascimento, Silva Dobora Dias da, Neri Anita Liberalesso, et al. (2013) Association between Underweight and Overweight/Obesity with Oral Health among Independently Living Brazilian Elderly. Nutrition, 29, 152-157. http://dx.doi.org/10.1016/j.nut.2012.05.011

[6] Mojon, P., Budtz-Jørgensen, E. and Rapin, C.H. (1999) Relationship between Oral Health and Nutrition in Very Old People. Age and Ageing, 28, 463-468. http://dx.doi.org/10.1093/ageing/28.5.463

[7] Lee, M.S., Huang, Y.C. and Wahlquist, M.L. (2010) Chewing Ability in Conjunction with Food Intake and Energy Status in Later Life Affects Survival in Taiwanese with the Metabolic Syndrome. JAGS, 58, 1072-1080. http://dx.doi.org/10.1111/j.1532-5415.2010.02870.x

[8] Liu, H., Maida, C.A., Spolsky, V.W., Shen, J., et al. (2010) Calibration of Self-Reported Oral Health to Clinically Determined Standards. Community Dentistry and Oral Epidemiology, 38, 527-539. http://dx.doi.org/10.1111/j.1600-0528.2010.00562.x

[9] Osterberg, T., Carlsson, G.E., Tsuga, K., et al. (1996) Associations between Self-Assessed Masticatory Ability and Some General Health Factors in as Swedish Population. Geroodentology, 13, 110-117. http://dx.doi.org/10.1111/j.1741-2358.1996.tb00162.x

[10] Eldre and Tandsundhed (2001) ÆldreForum 2001.

[11] Rentsch, A., Mojon, P., Roehrich, N., et al. (1995) The Orodental Situation of Aged Patients Hospitalized at the Hôpital de Gériatrie of Geneva. Schweizer Monatsschrift für Zahnmedizin, 105, 1523-1528. 